\title{
Observations of Different Type of Bursts Associated with M 6.3 Solar Flares
}

\author{
Z. S. Hamidi ${ }^{1, *}$, N. N. M. Shariff ${ }^{2, * *}$ \\ ${ }^{1}$ MARA Universiti of Technology, 40450, Shah Alam, Selangor, Malaysia \\ ${ }^{2}$ Department of Science and Technology Studies, University of Malaya, 50603, \\ Kuala Lumpur, Malaysia \\ *,**E-mail address: zetysh@salam.uitm.edu.my , nur.nafhatun@um.edu.my
}

\begin{abstract}
Variation of solar bursts due to solar flares such as type an isolated type III , a complex type III, $\mathrm{U}$ is being highlighted. These bursts occurred on $9^{\text {th }}$ March 2012 at the National Space Centre, Sg. Lang, Selangor, Malaysia Here, we study a unique case with a combination of two types burst associated with solar flare and CMEs. Our observation is focused on the low frequency region starting from $150 \mathrm{MHz}$ till $400 \mathrm{MHz}$. We found that a solar flare type solar flare type M 6.3 which occurred in active region AR 1429 starting from 3:32 UT and ending at 05:00 UT. The flare has been confirmed to be the largest flare since 2005. Some physical parameters will be measured. We then compared our results with X-ray data from NOAA Space Weather Prediction Centre (SWPC).
\end{abstract}

Keywords: Sun; low frequency; solar radio; burst, type III; type U; e-CALLISTO

\section{INTRODUCTION}

Sun is a unique and the closest star shines by converting protons into alpha particles. It is widely known that transportation of energy from the central regions of the Sun is primarily through photon radiation, although electron conduction contributes in the innermost region and convection dominates near the surface. Along the process, many research works have done to fulfil the objectives of this study. The dynamical behaviour of the Sun exhibits a variety of physical phenomena, some of which are still not at all or only barely understood sue to the complexity of the structure of the Sun.

In radio astronomy, solar radio emission provides an important diagnostic tool particularly suited for the analysis of non-thermal electron distributions, enhanced levels of various kinds of plasma waves and plasma phenomena related to electron acceleration in flares. The emission from solar flares is often called a radio burst that emphasizes the energetic and explosive characteristics of particles. Although the radio emission of a solar flare is much less energetic than flaring X- ray emission, solar bursts provide an important diagnostic tool for specifying the magnetic and temperature structures at a time as well as evidence for electrons accelerated to very high speed and powerful shock waves in radio region. It is well-known that studied on low-frequency solar radio bursts were amongst the first radio phenomena studied by radar scientists who turned their radio equipment to the skies after returning from World War II [1]. One main aspect of solar radio bursts at low 
frequencies lies in the fact that they originate in the same layers of the solar atmosphere in which geo-effective disturbances probably originate: the layers where energy is released in solar flares, where energetic particles are accelerated, and where Coronal Mass Ejections (CMEs) are launched.

The flares are classified of A,B, C, M or X according to the observed maximum X-Ray flux intensity and each successive class in an order of magnitude larger than previous. On 9th March 2012, solar flares are identified as M 6.3 class. Flares and CMEs lead to the generation of solar energetic particles (SEPs). The period began with a greater than $10 \mathrm{MeV}$ proton enhancement in progress. This increase was associated with a long-duration M2/1 n flare that occurred prior to the period and sourced from Region AR 1429. This flux enhancement continued until the onset if greater than $100 \mathrm{MeV}$ and greater than $10 \mathrm{MeV}$ early on 07th of March associated with flare at $07 / 0024 \mathrm{Z}$ and reached a maximum stage.

An extensive study of solar radio burst type III associated with solar flare event was carried out actively since 1958 [2]. During the explosion, these active regions relatively have strong magnetic fields with denser and hotter compared with their surroundings and enhanced radio burst typically from 10-100 minutes. Solar burst type III has been studied since the analysis of Clark Lake in 1962 [3] are thought to be produced by electron beams propagating along open magnetic field lines via the plasma emission mechanism. This type is one of the most studied solar coherent bursts, and generally occurs at the beginning of the impulsive phase of a solar flare. There are two steps process in which electrostatic oscillations are (i) first produced by the ejected solar flare via beam-plasma instability and (2) then converted to electrostatic oscillations, usually called Langmuir waves [4] are excited at frequencies near the local produced at both the fundamental $f_{p}$ and harmonic $2 f_{p}$.

Meanwhile, type II and type III solar radio bursts involve emission at two and only two harmonics [5,6]. In addition, type II bursts include a series of rapidly drifting bursts so reminiscent of type III bursts that they conjectured that the slow moving shock front was the seat of a continuous ejection of fast electrons. Some theoretical support for this view is provided by the acceleration mechanism. From the solar burst point of view, type III solar bursts are the most common types of bursts received by the solar corona associated with the solar flares which are characterized by a rapid drift from high to low frequency which is attributed to the decreasing electron plasma frequency fp encountered by the fast particles as they moved outward from the solar corona (Lin, 1981). These bursts were first discovered in the frequency range 500 to $10 \mathrm{MHz}$ [7] and then at lower frequencies using space radio instruments [8]. It was recently shown that a specific class of flares or Coronal Mass Ejections (CMEs) events associated with flare-generated shock waves exists For what appears on the surface to be an exotic mechanism, plasma emission is remarkably prevalent in the solar atmosphere, appearing in some form in quite a large fraction of flares [9] (Benz, 2005).This paper updates the largest solar flare that occurred on $9^{\text {th }}$ March 2012 and successfully detected in National Space Centre, Banting, Malaysia [10]. During that time, the solar flare almost produced a Coronal Mass Ejections (CMEs) and considered to be the largest flare since 2005 . Interestingly, during this event, three (3) solar bursts were successfully detected in the range of $150 \mathrm{MHz}$ till $384 \mathrm{MHz}$. Detailed discussion on this event will be described in the next section. Solar radio emission $U$ bursts with a change of the frequency drift sign are observed in a wide frequency band from decimeter to decameter waves [11-14]. The first observation of inverted type $U$ burst has been reported by [15]. Among the various impulsive of solar burst, type $U$ is a unique and interesting to study in detail in terms of association with solar flares. It is well understood that type $U$ burst is similar with a type III burst except the excited by 
electrons propagating in a magnetic arch. Based on previous studies, it occurs during the large flare, but still much more rarely and basically generated to invert of the J burst [16].

There is evidence that showed the most regions this type happen is between 20 to 200 $\mathrm{MHz}$ in asymmetry form [14]. Until now, the mechanism of generation of type $U$ bursts is still a matter of ongoing study. It was observed that in some cases, it will form in fundamental or harmonic structure but the configuration is very rare. Many theories have been proposed to explain type $U$ burst. Theoretically, there is an interpretation by [11] that suggested the electron beams propagating in closed coronal loops requires (i) a high ambient temperature in the relevant coronal loops which approximately $\mathrm{T}=7 \times 10^{6} \mathrm{~K}$ and (ii) the magnetic strengths should be higher compared with those implied by potential explorations. Electron beams traveling through the solar corona excite Langmuir waves near the local electron plasma frequency. When these Langmuir waves are converted into electromagnetic waves, the radiation can be detected in the radio region as bursts with very specific spectral signatures that depend on the magnetic structure which guides the beams. Normally, the ratio closed to open magnetic field structures increase with lower altitude Interestingly, it does not appear at a constant frequency but regularly decreasing turning frequency.

\section{METHODOLOGY}

In order to observe and record solar radio emission, Log Periodic Dipole Antenna (LPDA) was mounted on the top of the rooftop of National Space Centre (ANGKASA) building at Sg. Lang, Banting, Selangor located at (N 02 $\left.{ }^{\circ} 49.488^{\prime} \mathrm{E} 101^{\circ} 36.168^{\prime}\right)$ that covered the range of frequency from $85 \mathrm{MHz}$ till $470 \mathrm{MHz}$ [17]. This antenna is connected to the CALLISTO spectrometer via cable RG 58[18]. A preamplifier also is used to maximize the gain of the signal [19]. All the data are automatically saved in FIT files. On the other hand, due to constrain of the interference factors, the range of $150 \mathrm{MHz}$ till $350 \mathrm{MHz}$ had been chosen as selected data. Since the CALLISTO returns an output of a standard FITS file that represents a frequency versus time diagram, it is difficult to determine the types of solar flare. Thus it is necessary to process the image creating a specific diagram that gives a clear radio signature of flare or CMEs. We analysed using RAPP Java View based on the classification of metric and decimetric. The usual criteria taken into account were: bandwidth, duration, drift rate and substructures. We compare the result with data from GOES X-ray flux to determine the types of flare. The data were taken from NOAA/The Space Weather Prediction Center (SWPC) which provides solar activity forecast by the Geostationary Satellite System (GOES).

\section{RESULTS AND ANALYSIS}

Detection of solar burst type III has been observed on 9th March 2012 (about 3:32 UT till 5:00 UT) which is approximately occurring in 1 and half hour (88 minutes). We focused on the duration starting from the pre-stage of solar flare till the post stage of the flare. Associated with a type III burst is type U radio burst. 
2012/03/09 Radio flux density, e-CALLISTO (MALAYSIA), 3600 × 200 pixels

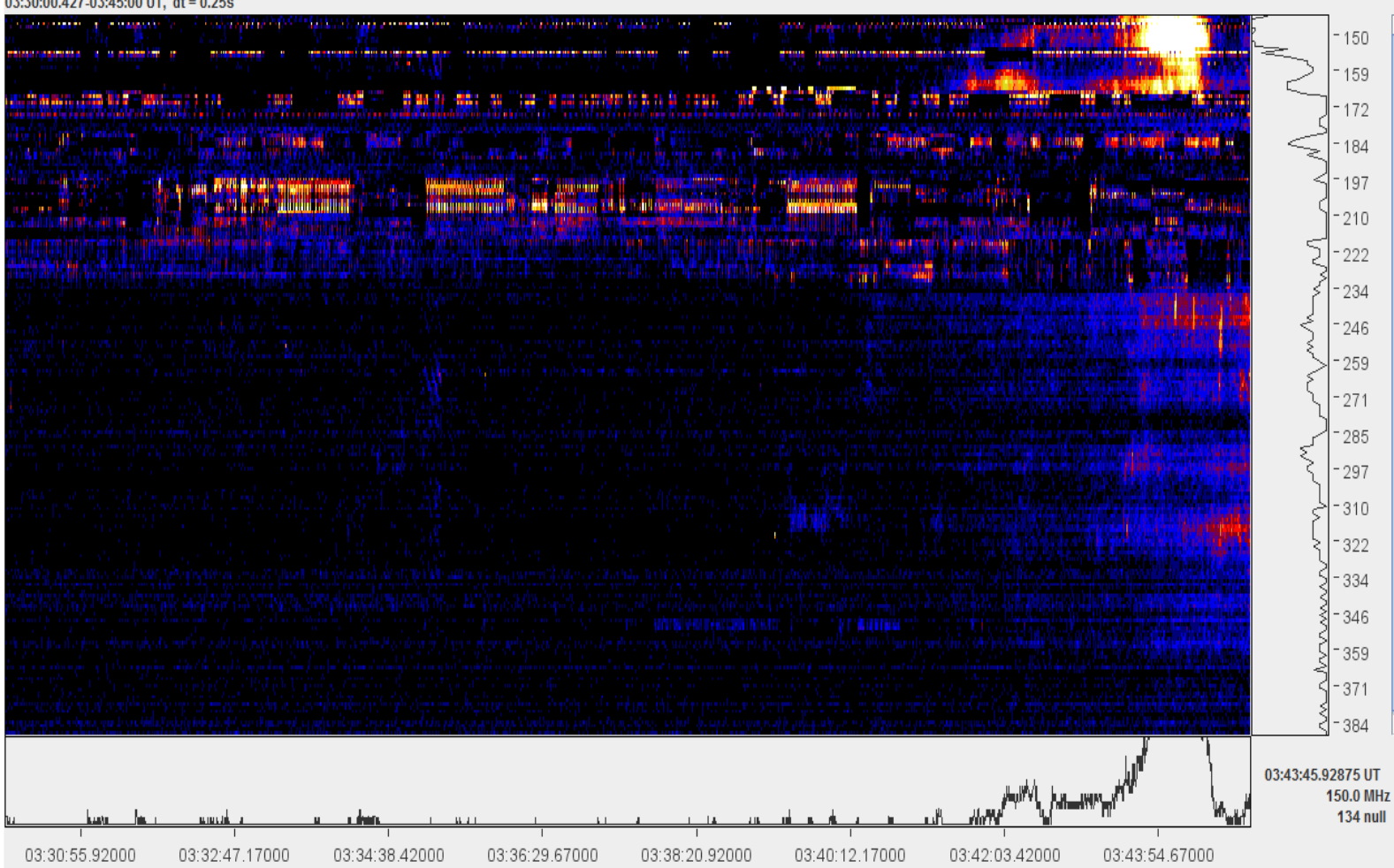

Figure 1. The pre- phase of solar burst.

201203:09 Radio flux density, e-CALUSTO (MALAYSIA), $3600 \times 200$ pixels 03:45:00.427-04:00:00 UT, dt - 0.25s

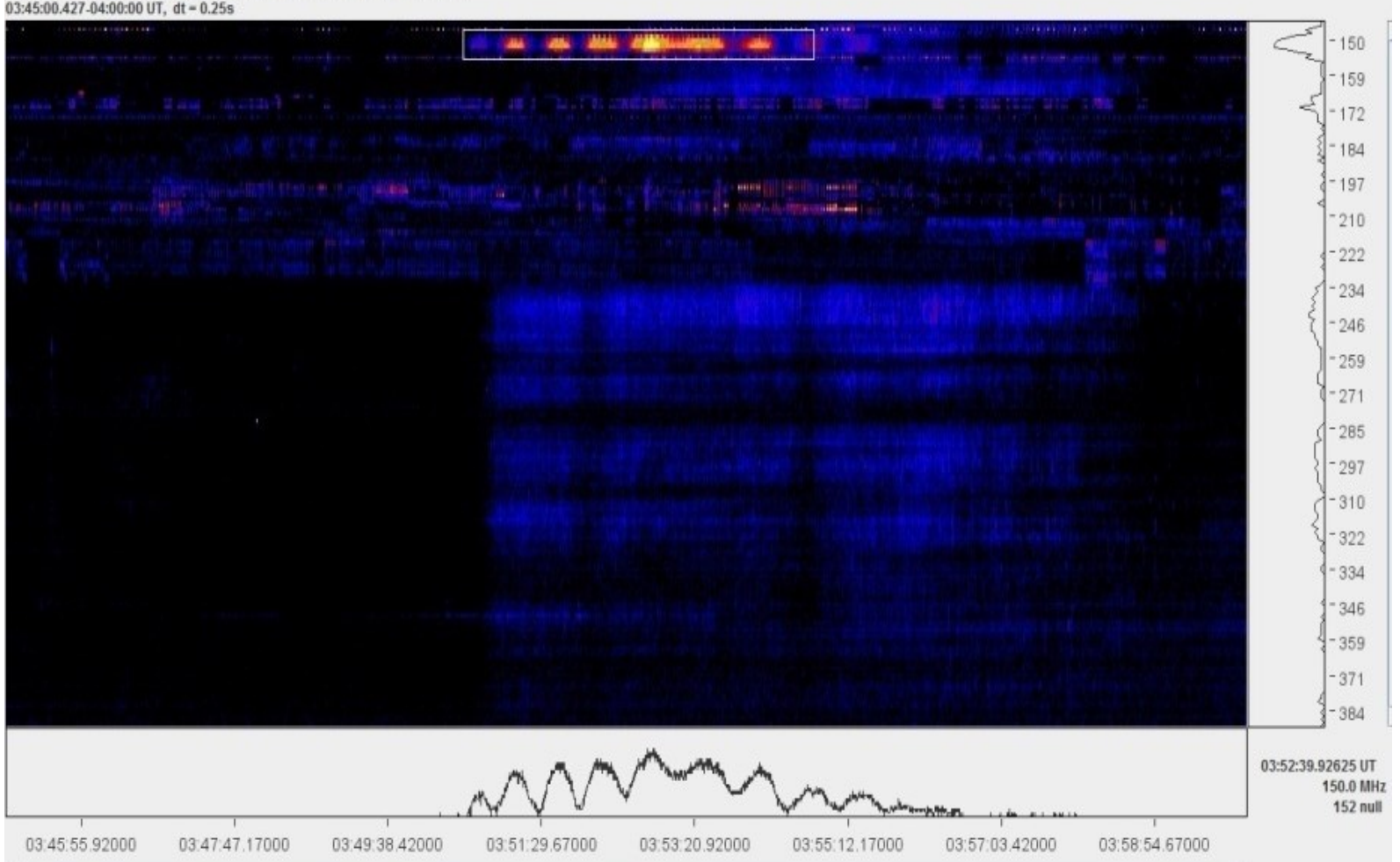

Figure 2. Significant continuum and complex solar burst type III. 
The beginning sign of a solar burst detected on 3: $41 \mathrm{UT}$ at $158-167 \mathrm{MHz}$ as shown in Figure 1. At 3:43 till 3:44 UT, the group of metric Type III bursts detected occurs at low frequency (150-164 MHz). This is an indicator that strong burst will occur due to the strong signal at the beginning of the phase.

We continue to analyze the next stage of this event. Between 3:45 till 3:58 UT, one could see that staring from 3:50UT, there is a strong background signal that covered the whole range of the spectra. As illustrated in Figure 2, the continuous radio burst was detected from 3:48 till 4:00 UT. At 3:50 till 3:55 UT on $150 \mathrm{MHz}$, a significant burst is detected and considered as type III burst.

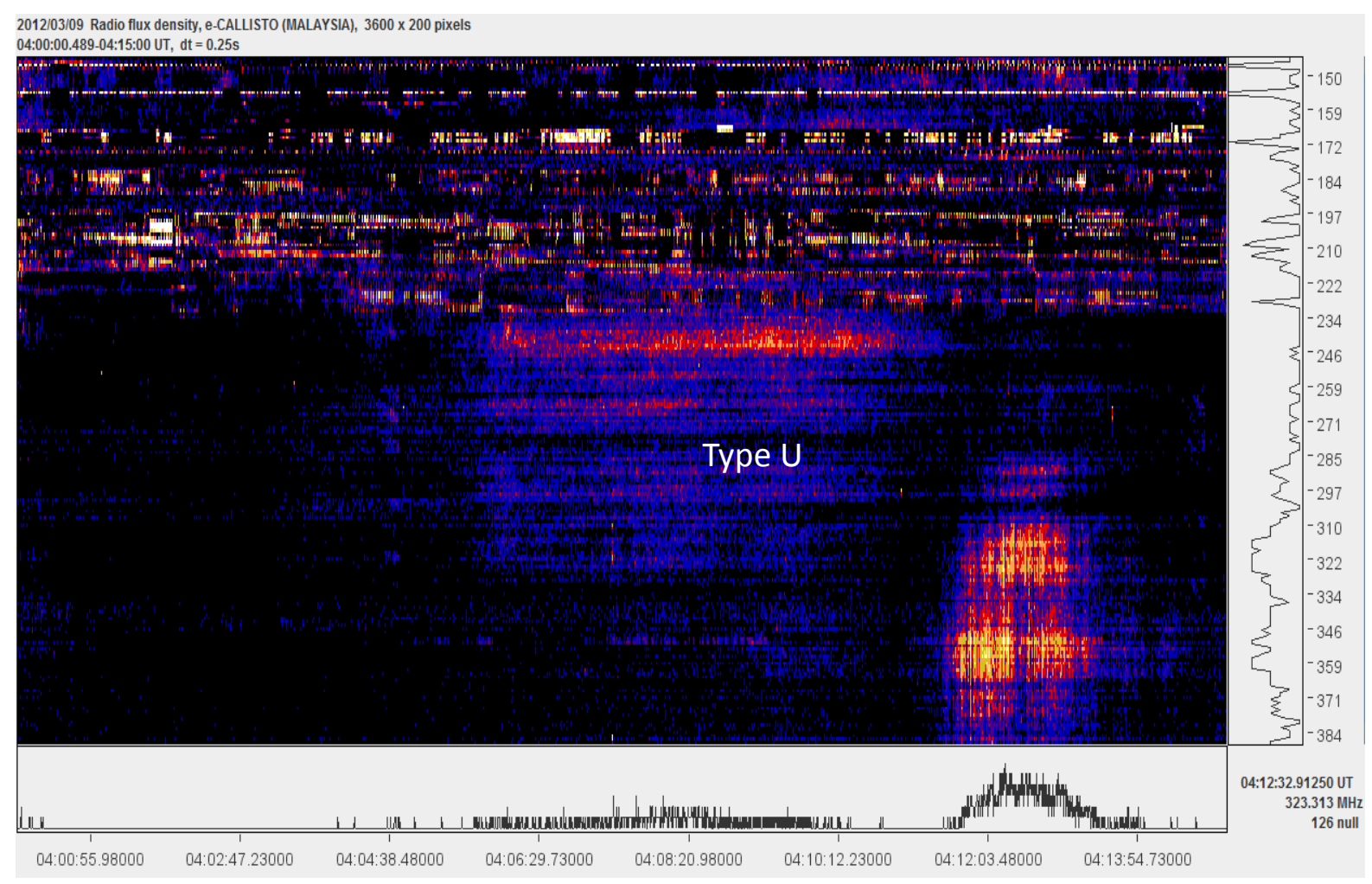

Figure 3. An inverted type U burst.

Interestingly, one could see solar burst type $U$ at 4:12 UT. This single type $U$ is formed from the continuous solar burst type III. A few active regions will produce type U burst and active region 1429 is the one of it. It is well known that this type is a sub-type of type III and the only difference between them is that the exciting electron beam of type $U$ follows a close loop instead of escaping from the Sun. This type is unique however, seldom occurred. In 2011 , there are only three (3) events were detected.

The maximum stage of solar flare and Coronal Mass Ejections can be observed within this range. In Figure 3, there is an isolated solar burst type III was detected at 4:22 UT. The duration of the smallest structures in decimetric burst generally decreases with increases frequency. The shock wave speed was calculated based on the spectrograph frequency versus time for the burst type II. From calculation, it understood that frequency drift (shock wave speed), $v_{1}>v_{2}$. This indicates that the electrons in $v_{1}$ move faster than electron in $v_{2}$. Thus, the speed of electrons at the beginning of Type II flares, $\mathrm{v}_{1}>1000 \mathrm{~km} \cdot \mathrm{s}^{-1}$ an evidence for shock in Coronal Mass Ejections (CMEs) or flare. 


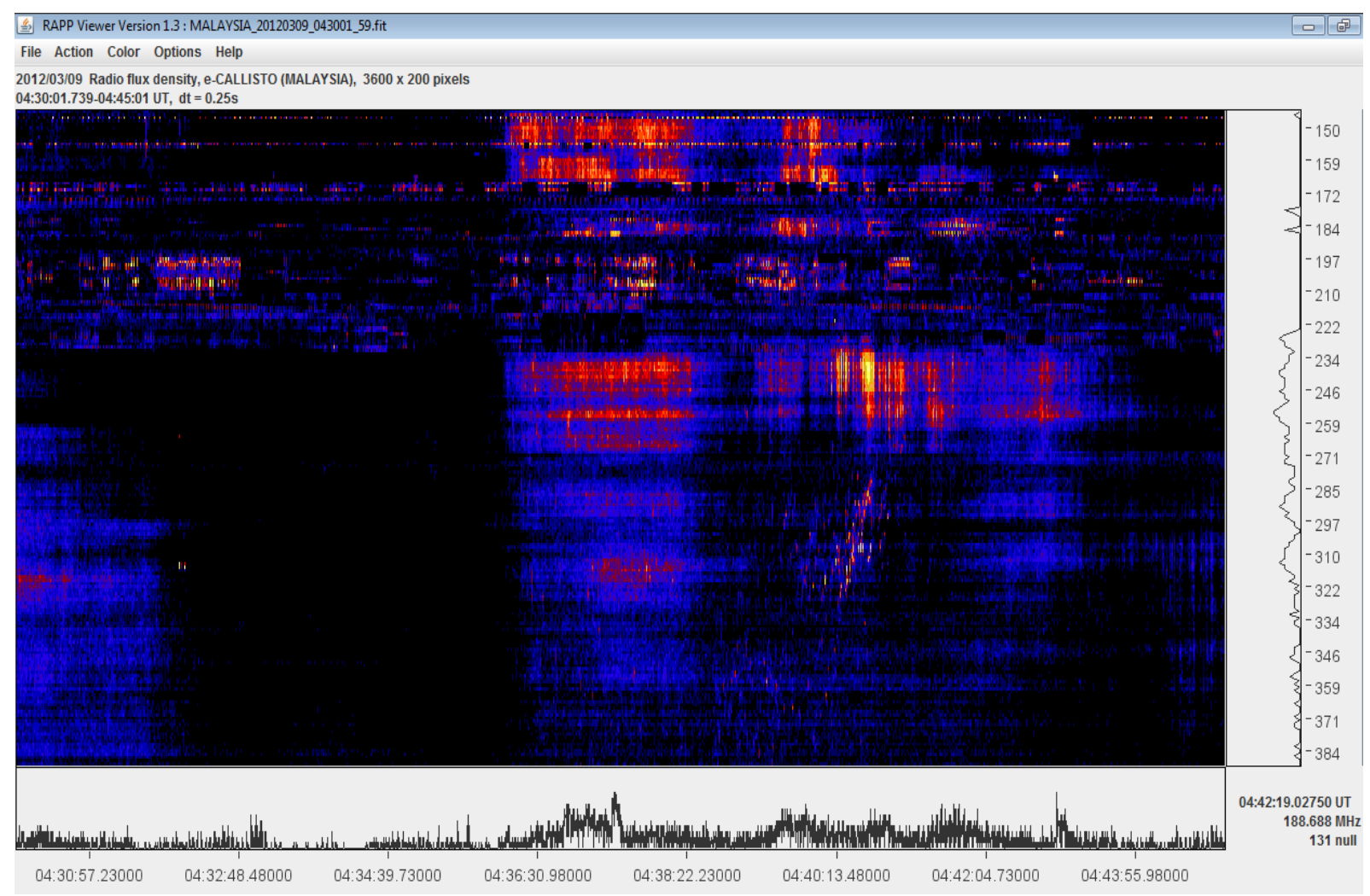

Figure 4. Continuous solar radio burst type III.

Fig. 4 illustrates the continuity of solar burst type III detected from 4:32 UT till 4:44 UT. From the figure, we expected that the solar burst slowly decrease and will be ending in a few minutes later. Type III can be considered as a domain type in this event due to the duration of the burst. Therefore, it is proved that type considered to be the relevant type associated with solar flares.

As a post stage of solar flare, we can observe the decreasing intensity of bursts including background signal. The radio flux intensity observed decreased till 4:45 UT. The background signal also back to normal. There is no burst detection in the end of 10 minutes.

\section{DISCUSSION AND CONCLUSION}

In general, for each figure showed a systematic variation of solar bursts type III with frequency from $150 \mathrm{MHz}$ till $400 \mathrm{MHz}$. Type III emission occurs in associated with solar flare M 6.3 classes which occurred in active region AR 1492 starting from 3:32 UT and ending at 05:00 UT.

Based on USAF/NOAA report of Solar and Geophysical Activity, a big sunspot in region AR 1429 which produced M6.3 at 09/0353Z peaking at 03:53 UT was large and magnetically complex during the period exhibiting a beta-gamma-delta magnetic configuration which appeared to slowly decay during the 9th- 10th of March 2012 but retained much of its complexity. In addition, associated with this event were type II (1285 $\mathrm{km} / \mathrm{s}$ ) and type IV radio sweeps along with a full halo coronal mass ejection (CMEs) which 
first seen in SOHO/LASCO C2 imagery at 09/0426Z. Velocity of CMEs recorded by STEREO B COR 2 imagery estimated to be travelling at $850 \mathrm{~km} / \mathrm{s}$.

In this paper we have presented observations of a results combination of solar bursts on 9th March 2012 All the three types (3) and U type of radio bursts are associated with solar flare and energetic CMEs, with the average speeds generally exceeding $1000 \mathrm{~km} / \mathrm{s}$. It has been suggested that the coverage of the radio region can be extended from $45 \mathrm{MHz}$ till 870 $\mathrm{MHz}$ to observe more drifting burst that might possibly occur in the next event. In short, it is confirmed that solar burst types III and U are the most relevant bursts with space weather condition. One should agree that solar radio monitoring will continue to play an important character in space weather studies due to wide region and sensitivity to the regions of the solar atmosphere especially in the corona and chromosphere in which many space weather phenomena originate. Our next task is to discuss the impact of solar bursts toward the Earth.

\section{Acknowledgement}

This work was partially supported by the PPP UM PV071/2011B grants. Special thanks to C. Monstein from ETH Zurich, Switzerland who set up and gives us training on analyzing the data. Also to National Space Agency and National Space Centre for giving us a site to set up this project and support this project. Solar burst monitoring is a project of cooperation between the Institute of Astronomy, ETH Zurich, and FHNW Windisch, Switzerland, MARA University of Technology and University of Malaya. This paper also used NOAA Space Weather Prediction Centre (SWPC) for the sunspot, radio flux and solar flare data for comparison purpose. The research has made use of the National Space Centre Facility and a part of an initiative of the International Space Weather Initiative (ISWI) program.

\section{Biography}

Zety Sharizat Hamidi is currently a $\mathrm{PhD}$ candidate and study in Solar Astrophysics specifically in radio astrophysics at the University of Malaya. Involve a project under the International Space Weather Initiative (ISWI) and also a lecturer in School of Physics and Material Science, at MARA University of Technology, Shah Alam Selangor.

N. N. M. Shariff Her current research is communicating sustainability. I'm looking forward for cross-field research i.e. solar astrophysics, light pollution measurement (mapping) and application of technology on sustainability.

\section{References}

[1] S. M. White, Asian Journal of Physics 16 (2007) 189-207.

[2] V. L. Ginzburg, V. V. Zheleznyakov, On the possible mechanisms of sporadic solar radio emission (radiation in an isotropic plasma), Sov. Astron. 2 (1958).

[3] W. C. Erickson, J. Geophys. Res. 68 (1963).

[4] T. A A. Langmuir, Oscillations in ionized gas, Physical Review 33 (1929).

[5] M. R. Kundu, Solar Radio Astronomy, John Wiley, 1965.

[6] J. P. Wild, J. Sci. Res 3 (1950a).

[7] J. P. Wild, Smerd S. F., Weiss A. A., Australian J. Sci. Res 12 (1959) 369.

[8] N. Gopalswamy, H. Xie, S. Yashiro, I. G., Usoskin, Coronal mass ejections and ground level enhancements, in: B.e.a. Sripathi Acharya (Ed.), 29th International Cosmic Ray Conference, Pune, India, 2005, pp. 169-172. 
[9] A. O. Benz, C. Monstein, A. H. Meyer, Solar Phys. 226 (2005) 143-151.

[10] Z. Hamidi, Z. Ibrahim, Z. Abidin, M. Maulud, N. Radzin, N. Hamzan, N. Anim, N. Shariff, Designing and Constructing Log Periodic Dipole Antenna to Monitor Solar Radio Burst: e-Callisto Space Weather, (2012).

[11] M. J. Aschwanden, Güdel M., ApJ 401 (1992).

[12] H. Aurass, Coronal Physics from Radio and Space Observations, in: I.G. Trottet (Ed.), Lecture Notes in Physics, Springer, Berlin, 1997.

[13] B. Kliem, M. Karlick'y, A. O. Benz, Astron. Astrophys. 360 (2000) 715-728.

[14] S. Suzuki, G. A. Dulk, Bursts of type III and type V, in: D. J. McLean, N. R. Labrum (Eds.), Solar Radiophysics: Studies of Emission from the Sun at Metre Wavelengths, Cambridge Univ. Press, New York, 1985.

[15] Swarup G., et.al, Astrophys. J. 131 (1960) 725.

[16] A. D. Fokker, Space Science Reviews 2 (1963) 70-90.

[17] Z. Hamidi, N. Shariff, Z. Abidin, Z. Ibrahim, C. Monstein, Middle-East Journal of Scientific Research 12 (2012) 6.

[18] Z. Hamidi, Z. Abidin, Z. Ibrahim, C. Monstein, N. Shariff, Signal Detection Performed by Log Periodic Dipole Antenna (LPDA) in Solar Monitoring, (2012).

[19] Z. Hamidi, S. Chumiran, A. Mohamad, N. Shariff, Z. Ibrahim, N. Radzin, N. Hamzan, N. Anim, A. Alias, American Journal of Modern Physics 2 (2013) 4. 